\title{
A FASE INICIAL DO PROCESSO DA VIVÊNCIA COM A DOENÇA DE PARKINSON
}

\section{THE INITIAL PHASE OF THE LIVING PROCESS WITH PARKINSON'S DISEASE}

\section{LA FASE INICIAL DEL PROCESO DE LA VIVENCIA CON LA ENFERMEDAD DE PARKINSON}

\author{
Rafaela Vivian Valcarenghi ${ }^{1}$, Angela Maria Alvarez ${ }^{2}$, Simony Fabíola Lopes Nunes ${ }^{3}$, \\ Andrelise Viana Rosa Tomasi ${ }^{4}$, Josiane Steil Siewert ${ }^{5}$, Rafaela Baptista ${ }^{6}$
}

\begin{abstract}
RESUMO
Objetivos: Conhecer a vivência da pessoa com doença de Parkinson desde o surgimento dos sinais e sintomas até a confirmação do diagnóstico. Métodos: Pesquisa qualitativa, utilizando o referencial metodológico da Teoria Fundamentada nos Dados. Participaram da entrevista em profundidade 30 pessoas com doença de Parkinson, cadastradas na Associação Parkinson Santa Catarina, e, posteriormente, cinco pessoas com a doença validaram os resultados. A coleta ocorreu entre setembro de 2013 e abril de 2014. Resultados: Emergiram três categorias: Percebendo que algo está mudando em suas capacidades, Percorrendo os consultórios médicos, e Lidando com o impacto da doença. Conclusão: Foi possível conhecer a vivência das pessoas com a doença em sua fase inicial, mostrando aspectos importantes na compreensão deste processo, e a partir de tal entendimento refletir sobre práticas de cuidados a esse grupo específico, incorporando assistência qualificada a esses usuários dos serviços de saúde.

Descritores: Doença de Parkinson; Doença crônica; Enfermagem; Cuidados de Enfermagem; Diagnóstico.
\end{abstract}

\begin{abstract} assistance to these users of the services Cheers.

\footnotetext{
${ }^{1}$ Centro Universitário Estácio de Santa Catarina

${ }^{2}$ Universidade Federal de Santa Catarina

${ }^{3}$ Universidade Federal de Santa Catarina

${ }^{4}$ Universidade Federal de Santa Catarina

${ }^{5}$ Universidade Federal de Santa Catarina

${ }^{6}$ Universidade Federal de Santa Catarina
}

Objectives: To know the experience of the person with Parkinson's disease from the appearance of the signs and symptoms to the confirmation of the diagnosis. Methods: Qualitative research, using the methodological framework of Data Based Theory. Participants of the in-depth interview were 30 people with Parkinson's disease, enrolled in the Santa Catarina Parkinson's Association, and later five people with the disease validated the results. The collection took place from September 2013 to April 2014. Results: There emerged three categories: Realizing that something is changing in their capacities, Going to physician offices, and Dealing with the impact of the disease. Conclusion: It was possible to know the experience of people with the disease in its initial phase, showing important aspects in the understanding of this process, and from that understanding reflect on care practices to this specific group, incorporating qualified

Descriptors: Parkinson disease; Chronic disease; Nursing; Nursing care; Diagnosis. 


\section{RESUMEN}

Objetivos: Conocer la vivencia de la persona con enfermedad de Parkinson desde el surgimiento de los signos y síntomas hasta la confirmación del diagnóstico. Métodos: Investigación cualitativa, utilizando el referencial metodológico de la Teoría Fundamentada en los Datos. En la entrevista en profundidad, 30 personas con enfermedad de Parkinson, registradas en la Asociación Parkinson Santa Catarina, y posteriormente cinco personas con la enfermedad validaron los resultados. La recolección ocurrió entre septiembre de 2013 y abril de 2014. Resultados: emergieron tres categorías: Percibiendo que algo está cambiando en sus capacidades, Recorrer los consultorios médicos, y Lidiando con el impacto de la enfermedad. Conclusión: Fue posible conocer la vivencia de las personas con la enfermedad en su fase inicial, mostrando aspectos importantes en la comprensión de este proceso, ya partir de tal entendimiento reflexionar sobre prácticas de cuidados a ese grupo específico, incorporando asistencia calificada a esos usuarios de los servicios de salud salud.

Descriptores: Enfermedad de Parkinson; Enfermedad crónica; Enfermería; Atención de Enfermería; Diagnóstico.

\section{INTRODUÇÃO}

As Doenças Crônicas Não transmissíveis (DCNTs) são as principais causas de mortes no mundo e têm gerado perda de qualidade de vida com alto grau de limitação nas atividades de trabalho e lazer. Apesar do rápido crescimento das DCNTs, seu impacto pode ser revertido por meio de intervenções de promoção de saúde para redução dos fatores de risco, além de melhoria da atenção à saúde, detecção precoce e tratamento oportuno. ${ }^{1}$

Os cuidados às pessoas em situação crônica são desafiadores para o enfermeiro, exigem diversos conhecimentos e habilidades. $^{2}$ Nesse sentido, uma das DCNTs que merece atenção por parte dos profissionais de saúde e de políticas públicas é a doença de Parkinson (DP) que acomete mais comumente pessoas com idade superior a 50 anos e sua incidência e prevalência tendem a aumentar com $\mathrm{o}$ avanço da idade. Por ser uma doença neurológica associada aos corpos de Lewy e perda de neurônios dopaminérgicos na substância negra, seus principais sinais são a bradiscinesia, rigidez, tremor de repouso e instabilidade postural. ${ }^{3}$

Conviver diariamente com dificuldades motoras gera grande estresse, uma vez que muitas atividades cotidianas requerem a execução de diferentes tarefas simultaneamente. ${ }^{4}$ Dessa forma, faz-se relevante que os enfermeiros tenham conhecimento sobre a vivência com tal condição, a fim de elaborarem intervenções adequadas para melhor convivência com a doença.

Este estudo tem como objetivo: Conhecer a vivência da pessoa com doença de Parkinson desde o surgimento dos sinais e sintomas até a confirmação do diagnóstico. 


\section{MÉTODO}

Trata-se de pesquisa qualitativa, que teve como Referencial Metodológico a Teoria Fundamentada nos Dados (TFD). A TFD está voltada para o conhecimento da percepção, do significado ou da maneira como as pessoas definem os eventos ou a realidade e como agem em relação às suas crenças. $^{5}$

Participaram do estudo 30 pessoas com DP cadastradas na Associação Parkinson Santa Catarina (APASC). Respeitou-se os preceitos da saturação teórica dos dados, realizando entrevistas em profundidade com três grupos amostrais, conforme o tempo de diagnóstico da doença: Grupo amostral 1 (tempo de diagnóstico da DP até 5 anos: 14 participantes); Grupo amostral 2 (tempo de diagnóstico da DP de 5 anos a 10 anos: 7 participantes) e Grupo amostral 3 (tempo de diagnóstico da DP acima de 10 anos: 9 participantes). No que diz respeito à idade dos participantes, variava de 44 anos a 83 anos. Os critérios de inclusão na pesquisa foram: ter diagnóstico da DP; condição cognitiva preservada, conforme escore do Mini Exame do Estado Mental (MEEM).

Os validadores dos resultados foram cinco pessoas que não participaram da primeira fase da pesquisa, com idades variando de 41 a 75 anos, e tempo de diagnóstico da DP entre dois e 18 anos.
A coleta de dados ocorreu de setembro de 2013 a abril de 2014. As entrevistas foram gravadas em mp3 e em seguida transcritas, para posterior análise e codificações de acordo com as técnicas estabelecidas pela TFD: codificação aberta (os dados são separados, examinados e comparados); codificação axial (as categorias são relacionadas e definidas suas subcategorias para gerar explicações precisas e completas sobre os fenômenos) e codificação seletiva (integra e refina as categorias). ${ }^{5}$

A pesquisa foi aprovada pelo Comitê de Ética em Pesquisa com Seres Humanos da Universidade Federal de Santa Catarina (UFSC), sob Parecer favorável número 329.662, CAAE: 14244513.9.0000.0121. Foi concedida autorização pela coordenação da APASC, para convidar as pessoas cadastradas. Os participantes assinaram o Termo de Consentimento Livre e Esclarecido (TCLE). Todas as fases desta pesquisa foram fundamentadas na Resolução 466/12 do Conselho Nacional de Saúde. ${ }^{6}$ Os participantes foram identificados com a letra "P" seguida de algarismo arábico.

\section{RESULTADOS}

A análise dos dados dos 30 participantes permitiu a revelação do fenômeno central: "Vivendo com a doença 
de Parkinson" e desvelou as categorias e subcategorias que compõem a fase inicial da doença, desde o surgimento dos sinais e sintomas até a confirmação do diagnóstico, como mostra a Figura 1:

Figura 1 - Modelo "Vivendo com a doença de Parkinson: A fase inicial do processo da vivência com a doença de Parkinson.”. Florianópolis, Santa Catarina, Brasil. 2015

\begin{tabular}{|c|c|c|}
\hline \multicolumn{3}{|c|}{ "Vivendo com a doença de Parkinson" } \\
\hline $\begin{array}{l}\text { PERCEBENDO QUE } \\
\text { ALGO ESTÁ } \\
\text { MUDANDO EM SUAS } \\
\text { CAPACIDADES }\end{array}$ & $\begin{array}{l}\text { PERCORRENDO OS } \\
\text { CONSULTÓRIOS } \\
\text { MÉDICOS }\end{array}$ & $\begin{array}{l}\text { LIDANDO COM O } \\
\text { IMPACTO DA } \\
\text { DOENÇA }\end{array}$ \\
\hline $\begin{array}{l}\text { "Tendo percepção do seu } \\
\text { estado de saúde" }\end{array}$ & $\begin{array}{l}\text { "Protelando a busca por } \\
\text { médico" }\end{array}$ & $\begin{array}{c}\text { subcategoria "Recebendo a } \\
\text { confirmação do } \\
\text { diagnóstico" }\end{array}$ \\
\hline $\begin{array}{l}\text { "Sendo alertado por } \\
\text { pessoas da família sobre o } \\
\text { comprometimento de suas } \\
\text { capacidades" }\end{array}$ & $\begin{array}{l}\text { "Recorrendo ao } \\
\text { atendimento médico". }\end{array}$ & "Revelando o diagnóstico" \\
\hline $\begin{array}{l}\text { "Sendo questionado por } \\
\text { outros sobre algo diferente } \\
\text { no seu comportamento" }\end{array}$ & $\begin{array}{l}\text { "Confirmando o } \\
\text { diagnóstico por meio de } \\
\text { médico especialista" }\end{array}$ & $\begin{array}{c}\text { "Vivendo o conflito da } \\
\text { aceitação" }\end{array}$ \\
\hline A fase inicial do $p$ & sso da vivência com a & ença de Parkinson \\
\hline
\end{tabular}

Fonte: própria das autoras, 2015.

Percebendo que algo está mudando em suas capacidades

Esta categoria retrata a forma como a pessoa começa a perceber que algo está modificando em seu viver, ou na sua condição de saúde. Esta percepção antecede o diagnóstico da doença, e tem período variado até a procura por serviços de saúde.

Nessa fase, sensações são compartilhadas com familiares ou pessoas próximas quanto às alterações relativas àquilo que os indivíduos e a comunidade consideram fora da normalidade. A percepção de que algo está mudando na sua saúde pode se revelar de várias maneiras, como mostram as subcategorias:

A subcategoria "Tendo percepção do seu estado de saúde" é evidenciada pelos principais sinais identificados pelas pessoas com DP, principalmente ao realizar atividades cotidianas:

[...] estava com dificuldade para enxugar o rosto, levantar objetos, escrever, tinha muita dificuldade para escrever [...] (P15)

[...] comecei a sentir muita fadiga, cansaço, e depois dessa fadiga eu tive perda de olfato. Dificuldade de escrita, por exemplo, um três ficava praticamente um zero, não conseguia fazer aquela curvinha... o tamanho da letra ia reduzindo [...] (P18) 
A percepção de que os movimentos estão diferentes, apresentando tremor ou rigidez, leva a pessoa a experimentar sentimentos diversificados. Alguns deles acreditavam que tais sinais e sintomas seriam passageiros, porém, quando começavam a persistir, sentiram a necessidade de descobrir o que estava acontecendo.

A subcategoria "Sendo alertado por pessoas da família sobre o comprometimento de suas capacidades" retrata o alerta por pessoas da família de que algo estava diferente com seu estado de saúde. É um momento importante, pois alguns até já haviam sentido algo estranho e agora estavam obtendo a confirmação feita pelos familiares. Por outro lado, em outras situações é o familiar que primeiramente observa que a pessoa está com algum tipo de alteração:

[...] as pessoas começaram a perceber, quando eu caminhava na praia, quando me movimentava... o problema da marcha, eu não levantava o lado direito do braço, o braço não acompanhava o movimento [...] (P18)

Meu filho falou assim: "Mãe, você está tremendo, para com isso!", ai olhei meu braço e falei: É verdade, mas não estou percebendo. Então aquele foi o primeiro momento que me dei conta ( $\mathrm{P} 12)$

A subcategoria "Sendo questionado por outros sobre algo diferente no seu comportamento" mostra que outras pessoas, como amigos, colegas de trabalho e desconhecidos, também notam alterações:

[...] outras pessoas que não eram ligadas a mim, até estranhos, perguntavam se eu tinha algum problema no braço, e pensei: tem alguma coisa mesmo, tem gente estranha notando (P25)

Nota-se um divisor de águas para a pessoa que inicia com sinais e sintomas ainda na sua fase produtiva. No decorrer da evolução da doença, colegas de trabalho começam a perceber dificuldades para realização de tarefas.

[...] falei com o meu patrão, ele falou: "Eu já sabia disso faz tempo!", pois eu tinha o sintoma... eu mexia com o pé, assim como se tivesse acelerando um carro... [...] Isso aí é o Parkinson mesmo, eu não queria falar para você, queria que você descobrisse." ...meu patrão [...] (P15)

[...] a parte cognitiva começou a ter problemas, de esquecer as coisas recentes, e um dia, num discurso, me perdi totalmente, desviei o foco... (P18)

As pessoas notam as alterações, significando que os sinais da DP começam a ser compartilhados com os outros. Tais alterações tornam-se ainda mais significativas para a pessoa na medida em que são transmitidas para a sociedade.

Percorrendo os consultórios médicos

Esta categoria sucede a percepção de que algo está mudando no estado de 
saúde. Alguns acreditam ser algo relacionado à condição de doença anterior, outros avaliam que possa ser a DP. As ideias relacionadas a possíveis diagnósticos fazem com que haja a procura por serviços de saúde, principalmente pelo profissional médico. Esse momento caracteriza-se como um itinerário utilizado pelas pessoas. Estas são encaminhadas de um médico para outro e para serviços de saúde. A seguir, são descritas as subcategorias:

A subcategoria "Protelando a busca por médico" demonstra o quanto as pessoas, mesmo percebendo sua condição, demoram a buscar apoio nos serviços de saúde. Elas julgam que pode ser em decorrência de outro problema de saúde ou procuram os serviços somente quando percebem uma evidência da condição. Buscar o médico é um comportamento esperado e compartilhado entre as pessoas, mesmo que elas resistam no primeiro momento:

[...] achei que esse pequeno tremor que começou depois de uns meses, poderia ser efeito colateral dessas injeções (que fazia pelas dores no braço), e logo eu não fui ao médico [...] (P16)

[...] vi que tinha alguma coisa errada, e fui deixando, deixando, até que fui no médico (P5)

$\mathrm{O}$ atendimento pelo profissional médico é o inicialmente procurado, como revelou-se na subcategoria "Recorrendo ao atendimento médico". As pessoas relatam a busca por um médico com quem já realizou algum tipo de tratamento anterior, alguém em quem se tenha confiança, para poder investigar esses sintomas.

Já a procura por médicos especialistas ocorre devido ao pensamento de possíveis outros diagnósticos, na espera que ao buscar o profissional médico seus problemas sejam rapidamente resolvidos, no entanto observa-se uma angústia nos depoimentos, ao perceberem que as soluções ou esclarecimentos não se dão imediatamente:

[...] fui num geriatra, porque fazia tempo que não ia a médico nenhum, aí fui fazer um checkup, para ver o que era, se era alguma falta de vitamina, ou alguma coisa que não estivesse bem, e ele mandou fazer alguns exames e depois diagnosticou na segunda consulta que era Parkinson (P16)

Fiz várias incursões a médicos, fui primeiro com meu clínico geral, que olhou e a primeira coisa que veio na cabeça: "Ah, é Parkinson, mas pela tua idade"... ele me pediu para fazer alguns testes, aí ele disse: “Talvez seja tremor essencial.”, e depois de alguns meses, como não passava, ele me encaminhou para um neurologista (P12)

Muitos médicos, através do exame clínico e/ou de exames complementares, sugerem tal diagnóstico e fazem encaminhamento a neurologistas para confirmação e tratamento.

A subcategoria "Confirmando o diagnóstico por meio de médico especialista" revelou que a procura pelo 
neurologista se dá de duas formas, por meio do encaminhamento por outro médico, que após exames de rotina identifica o possível diagnóstico, ou sugestão de outras pessoas para procura imediata pelo neurologista.

A confirmação do diagnóstico pelos neurologistas acontece da seguinte forma:

Me tratava para reumatismo, mas sempre aquele tratamento que a gente tomava remédio, mas não tinha efeito, aí em um dia da consulta a médica disse: "O teu caminhar não é de quem tem reumatismo, para mim é uma doença neurológica." Ela me encaminhou para um neurologista, isso em 1997, e desde então me trato. O neurologista logo diagnosticou” (P28)

A partir do neurologista ocorre a confirmação do diagnóstico, podendo gerar grande impacto na vida desta pessoa e sua família. Começa o tratamento específico para a DP, e surge, então, o momento de a pessoa interiorizar a condição e revelar à família e demais pessoas de seu convívio.

Lidando com o impacto da doença

Após a procura pelos serviços médicos, realização de exames de rotina e médicos especialistas, tem-se a confirmação do diagnóstico da doença. Por meio dos depoimentos dos participantes, pode-se notar que receber a confirmação da DP é bastante marcante, há vários sentimentos envolvidos, e estes fazem com que se torne mais ou menos difícil a revelação da condição para outras pessoas. Além disso, há o conflito em aceitar a nova condição de saúde.

$\mathrm{O}$ primeiro impacto ao receber o diagnóstico é visualizar uma condição de limitação associada às características da doença, revelado pela subcategoria "Recebendo a confirmação do diagnóstico". Devido ao senso comum do que seja a DP, isso faz com que a pessoa se imagine em condições de incapacidade e limitações. Por meio das falas é possível descrever a visualização carregada de impressões sobre imagens preconcebidas de um futuro com a doença e suas incapacidades:

O diagnóstico é terrível, bastante complicado, mesmo já tendo quase certeza que tinha Parkinson, mas na hora que recebe a gente perde o chão (P18)

Muitas pessoas relatam sentimentos de medo em relação às características da doença, sendo um impacto muito grande ao receber o diagnóstico:

[...] tive a impressão de que ia ficar dependente, que alguém teria que me cuidar, fiquei desesperada, porque, para quem faz tudo sozinha, se autodetermina, aí fiquei apavorada, para mim foi terrivel (P16)

Tinha medo porque não conhecia essa doença, via as pessoas tremer tanto, as pessoas que tinham essa doença, bem debilitada assim. Falei: Meu Deus, e agora? O que será de mim? Fiquei apavorada (P2) 
Por outro lado, alguns participantes conseguiram superar o impacto inicial do diagnóstico e visualizar sua nova condição de saúde com uma atitude resiliente.

[...] sou muito otimista, já levei para o lado do bem (P17)

[...] encarei com normalidade, não encarei como um fardo, sabia que ia ter limites e que era uma doença progressiva [...] (P19)

A subcategoria "Revelando o diagnóstico" descreve de que forma as pessoas, após o diagnóstico lidam com a revelação às demais pessoas de seu convívio. De acordo com os relatos, assumir-se com a doença e revelar sua condição a familiares e demais pessoas pode concorrer para certo empoderamento.

Apresentam-se os depoimentos de como se deu a revelação da doença:

[...] não fico só falando sobre a doença. Acho, que seja melhor para mim, não fico passando para os outros problemas de doença (P22)

[...] quem pergunta para mim eu digo, não tenho vergonha de dizer que tenho a doença, tem pessoas que escondem, que não gostam de dizer, mas eu não escondo de ninguém (P23)

[...] fiquei quatro meses sem contar para meus filhos, não tinha nem coragem de contar, e lá pelas tantas tive que dizer que estava com esse problema de saúde, mas foi muito difícil (P16)

Também se identificou que a revelação para outras pessoas significa querer assumir-se como alguém que continua vivendo normalmente, apesar da doença. Ao lidar com a doença, a pessoa tenta compartilhar seu problema com a comunidade e familiares, socializando informações e buscando a aceitação dos outros membros de seu grupo social.

A subcategoria "Vivendo o conflito da aceitação" mostra os significados de assumir todas as possibilidades que a doença carrega. A pessoa tenta retardar ao máximo essa interiorização como forma de resistência. A aceitação da condição de saúde varia de indivíduo para indivíduo, da mesma forma que o período de aceitação. Muitos levam tempo para aceitar que têm a doença, outros aceitam de forma um pouco mais tranquila, e outros ainda percebem a aceitação como uma imposição.

Identificam-se os conflitos que ocorrem até a aceitação da nova condição de saúde:

[...] está sendo muito difícil a aceitação, porque a gente duvida que seja, a medicação estava difícil de acertar, ainda estou trêmula, então fico sempre em dúvida, será que é mesmo? O remédio não está fazendo efeito por quê? Estou nessa fase (P6)

Tem dias que aceito, tem dias que não aceito (P10)

A convivência com DCNT engloba a aceitação e os efeitos sociais, culturais e as experiências das pessoas. Ter que aceitar é algo imposto, pois, a pessoa não tem escolha, por tratar-se de uma condição 
crônica. A DP condiciona as pessoas a terem que conviver com tal situação, e a aceitação influencia o modo como cada pessoa compreende e vivencia o dia a dia com a doença. Nos relatos podemos visualizar o sentimento de imposição frente à condição de cronicidade e a aceitação:

Estou tentando aceitar, porque não tem outra alternativa, não tem outro jeito, e estou tentando descobrir como é a melhor forma de conviver com essa situação (P16)

É difícil aceitar que alguém tem o Parkinson, e eu também, de uma certa forma não queria aceitar, não caía a ficha. Eu sei que não é uma doença terminal, mas sei que é uma doença difícil de ser lidada (P13)

A pessoa, ao descobrir a doença, passa por um processo de conflitos e momentos em que aceita a condição, em outros momentos não aceita a doença.

Esse conflito perdura por certo período, e com o decorrer do tempo a doença passa a ser superada, a pessoa vai percebendo que, embora tenha uma doença que pode ser limitante para as atividades, consegue conviver com a mesma:

Com o tempo se aprende a conviver com a doença, com as dificuldades (P16)

[...] foi bem sofrido, mas hoje não, hoje já levantei o astral (P5)

A forma como a situação é encarada faz com que se conviva da melhor forma:
Conviver com a doença primeiro é aceitar a condição, acho que não tem por que ficar questionamento muito o: porquê comigo? E quem não aceita, geralmente, percebo que alguns que não aceitam, sofrem mais (P18)

Desde o momento em que soube da doença, me atirei de forças e tudo, para poder vencer, e graças a esse meu esforço, fui bem-sucedida (P27)

Por meio da aceitação da doença é possível que as pessoas compartilhem atitudes e respostas positivas. As pessoas que acomodam a sua vida, passando por um processo de adaptação positivo encontram mais aceitação do grupo e sentem-se mais saudáveis.

\section{DISCUSSÃO}

O significado percebido pelos diferentes sinais e sintomas é susceptível de ser influenciado pela extensão com que eles interferem nos aspectos particulares da vida, alguns dos quais serão específicos para cada indivíduo, em especial atividades de ocupação e lazer. ${ }^{7}$ Isto implica em estar atento aos sinais e sintomas e ao adequado diagnóstico para dar início ao tratamento. ${ }^{8}$

A presença de sinais e sintomas decorrentes de uma doença crônica pode atuar como uma força que impulsiona familiares a se concentrarem de forma intensiva no cuidado com a pessoa em tal condição ${ }^{9}$, seja na tentativa de estarem atentos ao agravamento das alterações percebidas, ou ainda no apoio e estímulo. 
A categoria "Sendo questionado por outros sobre algo diferente no seu comportamento" mostra que, antes da procura por serviços médicos, as pessoas já haviam apresentado anteriormente algum sinal e sintoma que indicassem alguma desordem corporal. ${ }^{10}$

Protelar a busca pelos serviços médicos pode ser comparado aos dados de pesquisa sobre o tempo entre o início dos sintomas e o tratamento, onde os autores identificaram como as principais causas relatadas para demora em procurar atendimento médico: "achar que os sintomas não indicavam nenhuma doença" em 38,7\% dos casos; "custo para o deslocamento até uma unidade de saúde" em 16,1\%; "dificuldade em conseguir atendimento" em 14,6\%; "falta de tempo disponível para procurar uma unidade de saúde" em 12,6\%; e "achar que melhorariam espontaneamente" em $11,6 \% .^{11}$ Tais dados mostram a necessidade de serem compartilhadas com a sociedade em geral orientações sobre a DP, para que outras pessoas com os mesmos sinais e sintomas sejam estimuladas a procurar serviços de saúde para investigação, assim como os serviços de saúde devem estar preparados para fazer o diagnóstico e tratamento.

Em pesquisa com pessoas que utilizavam um centro para DP, os autores abordam sobre a otimização da assistência integral às pessoas com DP e a necessidade de verificação cuidadosa e periódica do tratamento. $^{12}$

O impacto do diagnóstico é o primeiro momento de dificuldade e que exige positiva superação para o bom convívio com a doença. Existe ainda falta de informação quanto ao diagnóstico, tanto por parte profissional, quanto para a comunidade, atrasando desta forma o início do tratamento. ${ }^{(10)}$ É muito difícil receber o diagnóstico por afetar aspectos físicos, emocionais, mentais e sociais de suas vidas e também de seus familiares. ${ }^{13}$

Os sentimentos diante do diagnóstico mostram-se importantes para os enfermeiros, para que assistam as pessoas com DP e seus familiares através de orientação, apoio, manejo dos sintomas e planejamento dos cuidados. ${ }^{14}$

Os diálogos e interações que propiciam a revelação do diagnóstico devem ser estimulados pelos profissionais de saúde, reforçando o enfrentamento e o empoderamento das pessoas no sentido de combater preconceitos e estigmas da doença.

Nesse sentido, entende-se a importância da equipe multiprofissional de saúde em estabelecer um vínculo, através da comunicação efetiva entre o indivíduo com a DP e o seu cuidador familiar. ${ }^{15}$ 
Observa-se certa negação da doença, aspecto relevante no processo da vivência com a DP. A aceitação é considerada um processo interno no qual a pessoa se reconhece e assume sua realidade. O processo de aceitação é influenciado principalmente pela "maneira de olhar" das pessoas, ou seja, há pessoas que negam a doença ou têm mais dificuldade em aceitar do que aqueles que conhecem e têm um olhar positivo sobre a situação. ${ }^{16}$

\section{CONCLUSÃO}

Foi possível conhecer como ocorre a vivência com a DP desde a fase inicial, dos primeiros sinais e sintomas até a aceitação da condição. A pessoa pode se perceber com alguma alteração, bem como pessoas da família, amigos, colegas de trabalho podem notar alguma modificação. Após tal percepção, inicia-se um itinerário em busca de profissionais médicos para investigação, que pode ser adiado, contudo acaba na busca a várias especialidades, até a confirmação feita pelo médico neurologista.

Quanto às limitações, nesta pesquisa os participantes deste estudo foram auto selecionados e, embora representativos da população geral de pessoas com DP no Brasil, suas percepções podem não refletir a população geral de pessoas com DP que vivenciam a doença em outros contextos culturais, entretanto, a compreensão detalhada desses participantes e $\mathrm{o}$ alinhamento com os achados de outros estudos se somam ao corpo de conhecimento nesse campo.

O estudo fornece informações relevantes que poderão influenciar no modo de produção do cuidado às pessoas com DP. E ainda, tem como contribuições o conhecimento da vivência com a DP, para que os enfermeiros estejam atentos não só às características motoras da doença, mas aos sentimentos e necessidade de cada pessoa. A enfermagem tem papel primordial no vínculo e assistência à pessoa com DP e sua família, por meio de atividades que promovam saúde por meio de um viver saudável.

\section{REFERÊNCIAS}

1. Ministério da Saúde. Brasil. Plano de ações estratégicas para o enfrentamento das doenças crônicas não transmissíveis (DCNT) no Brasil: 2011-2022.

Departamento de Análise de Situação de Saúde. - Brasília: Ministério da Saúde, 2011, 148 p.

2.Furtado LG, Nóbrega MML. Model of care in chronic disease: inclusion of a theory of nursing. Texto contexto - enferm. [Internet]. 2013 Dez [cited 2017 May 26]; 22(4): 1197-1204. Available from: http://www.scielo.br/scielo.php?script=sci _arttext\&pid=S0104-07072013000400039 3. Kalia L. Parkinson's disease. The Lancet. 2015; 386 (9996): 896-912. 4. Terra MB, Rosa PC, Torrecilha LA, Costa BT, Ferraz HB, Santos SMS. Impacto da doença de Parkinson na performance do equilíbrio em diferentes demandas atencionais. Fisioter. Pesqui. 
[Internet]. 2016 Dec [cited 2017 May 26]; 23(4):410-415. Available from: http://www.scielo.br/scielo.php?script=sci _arttext\&pid=S180929502016000400410\&lng=pt\&nrm=iso 5. Strauss, A, Corbin J. Pesquisa qualitativa: técnicas e procedimentos para o desenvolvimento de teoria fundamentada. $2^{\mathrm{a}}$ ed. Porto Alegre (RS): Artmed, 2008.

6. Brasil. Resolução nº 466/2012. Dispõe sobre as diretrizes e normas regulamentadoras de pesquisas envolvendo seres humanos. Brasília: Conselho Nacional de Saúde; 2012.

7. Reddy P, Martinez-Martin P, Brown RG, Chaudhuri KR, Lin JP, Selway R, et al. Perceptions of symptoms and expectations of advanced therapy for Parkinson's disease: preliminary report of a Patient-Reported Outcome tool for Advanced Parkinson's disease (PROAPD). Health and Quality of Life Outcomes. [Internet]. 2014 [cited 2016 Dec 26]; 12(11):1-10. Available from: https://www.ncbi.nlm.nih.gov/pmc/articles /PMC3906878/

8. Ju YS, Habermann B. Nursing Research in Parkinson's Disease From 2006 to 2015 A Systematic Review. Clinical Nursing Research. 2017 Apr; 26(2):142-156. 9. Chen T, Thomas C, Habermann B, Berger S, Tickle-Degnen L. Through the Eyes of the Care Partner: Daily Experiences Living With a Person With Parkinson's Disease. American Journal of Occupational Therapy, 2016;

70(4_Supplement_1).

10. Sorrell J. Living with Parkinson's Disease. J Psychosoc Nurs Ment Health Serv [Internet]. 2017 Apr [cited 2017 May 26]; 55(4):15-18. Available from: https://www.ncbi.nlm.nih.gov/pubmed/284 07154

11. Maior ML, Guerra RL, CailleauxCezar M, Golub JE, Conde MB. Tempo entre o início dos sintomas e o tratamento de tuberculose pulmonar em um município com elevada incidência da doença. J. bras. pneumol. [Internet]. 2012 [cited 2016 Dec
26]; 38(2): 202-209. Available from: http://www.scielo.br/scielo.php?script=sci _arttext\&pid=S1806-

37132012000200009\&lng=en.

12. Kessler D, Liddy C. Self-management support programs for persons with Parkinson's disease: An integrative review. Patient Educ Couns [Internet]. 2017 Apr [cited 2017 May 20]; S0738-3991(17): 30244-6. Available from: http://www.pecjournal.com/article/S0738-3991(17)302446/fulltext

13. Soleimani M, Bastani F, Negarandeh R, Greysen R. Perceptions of people living with Parkinson's disease: a qualitative study in Iran. Br J Community Nurs [Internet]. 2016 Apr [cited 2017 May 26]; 21(4):188-195. Available from: http://www.magonlinelibrary.com/doi/full/ 10.12968/bjcn.2016.21.4.188 14. Galvão TLA, Oliveira KKD, Maia CAAS, Miranda FAN. Assistance to the person with Parkinson carrier in the context of family health strategy Rev. pesqui. cuid. fundam. [Internet]. 2016 Dec. [cited 2017 May 26]; 8(4): 5101-5107 Available from:

http://www.seer.unirio.br/index.php/cuidad ofundamental/article/view/4448/pdf_1

15. Ferreira DPC, Coriolano MGWS, Lins CCSA. Knowledge as a tool to promote care of the elderly with Parkinson's disease. Rev. enferm. UFPE on line[Internet]. 2016 Dec [cited 2017 May 26]; 10(12):4628-4636. Available from: http://www.revista.ufpe.br/revistaenfermag em/index.php/revista/article/viewArticle/9 830

16. Zaragoza SA, Senosiain GJM, Riverol FM, Anaut BS, Díaz SCA, Ursúa MES. et al. Key elements in the process of living with Parkinson's disease for patiens and caregivers. Anales Sis San Navarra [Internet]. 2014 Apr [cited 2017 May 26]; 37(1):69-80. Available from: http://scielo.isciii.es/scielo.php?script=sci_ arttext\&pid=S113766272014000100008\&lng=es 
RECEBIDO: 07/12/18

APROVADO: $12 / 03 / 19$

PUBLICADO: 07/19 